\title{
EPIDEMIOLOGIA DAS HOSPITALIZAÇÕES POR DIARREIA E GASTROENTERITE EM UM HOSPITAL UNIVERSITÁRIO PEDIÁTRICO
}

\author{
EPIDEMIOLOGY OF HOSPITALIZATIONS BY DIARRHEA AND \\ GASTROENTERITIS IN A UNIVERSITY PEDIATRIC HOSPITAL
}

\author{
Alex de Novais Batista ${ }^{1}$ \\ Letícia Pinheiro de Melo ${ }^{2}$ \\ Thales José Nunes Vieira ${ }^{3}$ \\ Geofabio Sucupira Casimiro ${ }^{4}$
}

Kennia Sibelly Marques de Abrantes ${ }^{5}$

RESUMO: Objetivo: Identificar o perfil epidemiológico das hospitalizações em crianças e adolescentes por diarreia e gastroenterite ocorridas no período de 01 de janeiro de 2014 a 31 de dezembro de 2018 no Hospital Universitário Júlio Bandeira (HUJB), localizado na cidade de Cajazeiras-PB. Métodos: Trata-se de estudo ecológico, transversal, exploratório e descritivo, de abordagem quantitativa, de base documental e de campo. A coleta de dados ocorreu por meio da consulta aos prontuários de pacientes de 0 a 19 anos, hospitalizados por diarreia ou gastroenterite no período compreendido entre janeiro de 2014 a dezembro de 2018. Após a coleta, as informações foram importadas para o programa estatístico IBM SPSS Statistics 21 para realização de análises estatísticas descritivas, sendo também calculada a proporção de hospitalizações por diarreia e gastroenterite. Resultados: Houve um total de 334 internamentos por diarreia e gastroenterite no HUJB na população estudada durante o período determinado para a pesquisa, com redução numérica de $29,4 \%$ se comparados com os dados referentes ao ano inicial e ao ano final da pesquisa. Uma a cada quatro hospitalizações no mês de fevereiro ocorreu por diarreia e gastroenterite e os maiores números concentraram-se em crianças menores de 5 anos $(73,1 \%)$, cor/raça parda $(57,8 \%)$, moradores da zona urbana $(69,5 \%)$ e procedentes do município de Cajazeiras-PB $(56,9 \%)$. Conclusão:

\footnotetext{
1 Graduando do curso de Medicina da UFCG - Campus Cajazeiras. E-mail: alexnovaisb@gmail.com.

2 Graduando do curso de Medicina da UFCG - Campus Cajazeiras. E-mail: leticia.pinheiro.melo@gmail.com.

${ }^{3}$ Graduando do curso de Medicina da UFCG - Campus Cajazeiras. E-mail: thalesjnvieira@gmail.com.

${ }^{4}$ Farmacêutico-Bioquímico, Mestre em Sistemas Agroindustriais, Professor do curso de Enfermagem e Medicina da UFCG - Campus Cajazeiras. E-mail: geosucupira@gmail.com.

5 Enfermeira, Doutora em Ciências da Saúde, Professora do curso de Enfermagem da UFCG Campus Cajazeiras. E-mail: kenniaabrantes@gmail.com.
} 
A partir da identificação da distribuição das hospitalizações ao longo dos meses do ano e do perfil populacional mais afetado por essas afecções é possível obter melhor planejamento da instituição de saúde a fim de garantir insumos necessários às demandas específicas do tratamento, assim como realizar treinamento profissional e promover educação em saúde da comunidade.

Palavras chave: Diarreia. Gastroenterite. Hospitalização. Criança. Adolescente.

ABSTRACT: Objective: To identify the epidemiological profile of hospitalizations in children and adolescents due to diarrhea and gastroenteritis, at the University Hospital Júlio Bandeira (HUJB), located in the city of Cajazeiras-PB, which took place from January $1^{\text {st }}$ of 2014 to December 31st of 2018. Methods: This is an ecological, transversal, exploratory and descriptive study, with a quantitative approach, based on documents and in the field. Data collection occurred by consulting the medical records of patients aged 0 to 19 years hospitalized for diarrhea or gastroenteritis in the period between January 2014 and December 2018. After the collection, the information was imported into the program IBM SPSS 21 to carry out descriptive statistical analyzes, and the proportion of hospitalizations due to diarrhea and gastroenteritis was also calculated. Results: There were a total of 334 hospitalizations for diarrhea and HUJB gastroenteritis in this population and the respective period of time, with a numerical reduction of $29,4 \%$ when comparing the data referring to the initial and final years of the research. One in four hospitalizations in February occurred due to diarrhea and gastroenteritis and the largest numbers were concentrated in children under 5 years old $(73,1 \%)$, color/brown race $(57,8 \%)$, residents of the urban area (69,5\%) and from the municipality of Cajazeiras-PB (56,9\%). Conclusion: Through the identification of the distribution of hospitalizations over the months of the year and the population profile most affected by these conditions, it is possible to obtain a better planning of the health institution in order to guarantee basic supplies to the specific demands of the treatment, as well as to carry out professional training and promote community health education.

Keywords: Diarrhea, Gastroenteritis, Hospitalization, Child, Adolescent. 


\section{INTRODUÇÃO}

A diarreia consiste numa manifestação comum de doenças infecciosas intestinais e pode ser definida como a ocorrência de três ou mais evacuações de fezes com consistência líquida ou aquosa em um período de 24 horas (WIELGOS et al., 2019). Segundo Araújo (2010), as principais complicações deste quadro são a desidratação, o desequilíbrio hidroeletrolítico e a desnutrição, que podem levar o paciente a óbito caso não seja instituído o devido tratamento.

Em relação à sua epidemiologia mundial, as diarreias e gastroenterites são cotadas como a segunda principal causa de mortalidade infantil, representando cerca de $9 \%$ dos óbitos mundiais nessa população. Além do mais, estimativas indicam que ainda ocorram cerca de 2,5 bilhões de casos de doença diarreica por ano em crianças menores que cinco anos de idade, que geram como consequência uma média de 1.400 óbitos diários (PINZON-RONDÓN et al., 2015).

Diversos patógenos estão relacionados com a sua etiologia, como vírus, bactérias e parasitas. Dentre os seus mecanismos de trasmissão destacam-se via fecal-oral, contato interpessoal ou através de água, alimentos ou objetos contaminados, o que torna a ausência de condições sanitárias e de medidas adequadas de higiene os principais fatores relacionados ao desenvolvimento de diarreias e gastroenterites (ANDERS et al., 2015).

Nas últimas décadas, os números nacionais de morbimortalidade devido às doenças diarreicas apresentaram redução significativa, por fatores como melhoria dos indicadores nacionais socioeconômicos, demograficos e de saúde. Entretanto, de acordo com Bülher (2014), essas afecções continuam representando um grande problema de sáude pública nacional, pois as regiões Norte e Nordeste continuam a concentrar os maiores números de internações e de óbitos do país, ainda devido à presença de piores indicadores sociais e de saúde.

Em relação à região Nordeste, além dos fatores já citados, as características climáticas também corroboram para a elevada ocorrência de diarreias e 
gastroenterites, pois em períodos chuvosos a água superficial acaba arrastanto dejeitos que podem contaminar as fontes de água utilizadas pela população, já em períodos de seca, ocorre maior utilização de água provinda de fontes alternativas e qualidade duvidosa (PAZ; ALMEIDA; GÜNTHER, 2012).

Dessa forma, ao considerar diarreias e gastroenterites como sérios problemas de saúde pública nacional e especialmente para a Região Nordeste, a presente pesquisa objetivou identificar o perfil epidemiológico de hospitalizações de crianças e adolescentes em decorrência dessas patologias, em um Hospital Universitário localizado no alto sertão do estado da Paraíba, cidade de Cajazeiras, no período de 01 de janeiro de 2014 a 31 de dezembro de 2018.

\section{MATERIAL E MÉTODOS}

Esta pesquisa foi estruturada como um estudo ecológico, transversal, exploratório e descritivo, foi adotada uma abordagem quantitativa, de base documental e de campo. O local do estudo consistiu-se no Hospital Universitário Júlio Bandeira-HUJB, o qual é referência no atendimento pediátrico de média complexidade para a área que polariza todos os municípios que compõem a $9^{a}$ Região de Saúde da Paraíba, a qual envolve 15 municípios, aproximadamente 175.657 habitantes, sendo Cajazeiras o município sede (SILVA et al., 2019).

Para tanto, a fonte de dados consistiu nos prontuários de internamentos de crianças e adolescentes de 0 a 19 anos, de acordo com a classificação da World Heath Organization-WHO (1995), internados por diarreia e gastroenterite no período de 01 janeiro de 2014 a 31 de dezembro de 2018, identificadas por ocasião do seu internamento, através do registro e preenchimento da Autorização de Internação Hospitalar (AlH). Para a coleta das informações, elaborou-se um instrumento com questões inerentes aos pacientes baseando-se nas informações contidas nos prontuários médicos.

Nos prontuários, a página utilizada para coleta de dados restringiu-se à $\mathrm{AlH}$ e as variáveis incluídas foram: ano e mês do internamento, município de residência, 
zona de residência, sexo, idade e cor/raça. Os dados foram coletados por meio da técnica de pesquisa documental indireta, em instrumento específico, por um único pesquisador e os critérios de inclusão estabelecidos foram todos os internamentos ocorridos no período investigado, cuja hipótese diagnóstica na AlH refere-se à diarreia, gastroenterite ou doença infecciosa intestinal, que respondem aos objetivos propostos do estudo, não havendo critérios de exclusão.

Após a coleta, as respostas foram importadas para o programa estatístico IBM SPSS Statistics 21 para realização de análises estatísticas descritivas (distribuições absolutas, porcentuais, média, moda e desvio padrão).

Ressalta-se que foram obedecidos todos os itens dispostos na Resolução 466/12 do Conselho Nacional de Saúde, que regulamenta a pesquisa com seres humanos. Esta pesquisa foi submetida ao Comitê de Ética em Pesquisa do Centro de Formação de Professores da Universidade Federal de Campina Grande, para apreciação e parecer, sendo aprovada com o número do parecer 2.672.468.

\section{RESULTADOS}

Houve um total de 334 internamentos por diarreia e gastroenterite no Hospital Universitário Júlio Bandeira, no período 01 de janeiro de 2014 a 31 dezembro de 2018. Na Tabela 1 abaixo, observa-se a distribuição numérica dos internamentos por diarreia e gastroenterite e a respectiva proporção que representaram em relação às demais causas de internamento no serviço. 
Tabela 1 - Distribuição do número de internamentos por diarreia e gastroenterite e proporção de hospitalizações por diarreia e gastroenterite, por ano. Hospital Universitário Júlio Bandeira, Cajazeiras-PB, 2014-2018.

\begin{tabular}{lccccccc}
\hline Anos & $\mathbf{2 0 1 4}$ & $\mathbf{2 0 1 5}$ & $\mathbf{2 0 1 6}$ & $\mathbf{2 0 1 7}$ & $\mathbf{2 0 1 8}$ & Total \\
\hline $\begin{array}{l}\text { No total de internamentos no } \\
\text { serviço }\end{array}$ & 478 & 421 & 514 & 417 & 615 & 2.445 \\
$\begin{array}{l}\text { № de internações por diarreia e } \\
\text { gastroenterite }\end{array}$ & 85 & 60 & 84 & 45 & 60 & 334 \\
$\begin{array}{l}\text { Proporção das hospitalizações por } \\
\text { diarreia e gastroenterite (\%) }\end{array}$ & 17,8 & 14,3 & 16,3 & 10,8 & 9,8 & 13,8 \\
\hline
\end{tabular}

Fonte: Prontuário de Internamento. Hospital Universitário Júlio Bandeira, CajazeirasPB: 01 de janeiro de 2014 a 31 de dezembro de 2018.

De acordo com a Tabela 1, observa-se que o ano de 2014 destacou-se por concentrar o maior número e proporção de internações devido a essa causa, além do mais, ao comparar-se os números referentes ao ano inicial e ao ano final da pesquisa, houve uma redução numérica dos internamentos por diarreia e gastroenterite de $29,4 \%$ e da proporção de hospitalizações por diarreia e gastroenterite de 44,9\%.

A maioria dos registros hospitalares ocorreu no primeiro semestre do ano, uma vez que até o mês de junho já se somava um quantitativo de $58,1 \%$ do total de internações. Outro ponto importante é que apesar do mês de março ter concentrado o maior número absoluto de hospitalizações no período ( $41 ; 12,3 \%$ ), o mês de fevereiro destacou-se por deter a maior proporção dessas hospitalizações no serviço, de $26,3 \%$, ou seja, aproximadamente 1 a cada 4 admissões neste mês ocorreu por diarreia e gastroenterite. O Gráfico 1 abaixo reúne dados referentes à distribuição do número de internamentos devido à diarreia e gastroenterite de acordo com os meses do ano e sua respectiva proporção. 
Gráfico 1 - Distribuição do número de internamentos e proporção de hospitalizações por diarreia e gastroenterite, por meses do ano. Hospital Universitário Júlio Bandeira, Cajazeiras-PB, 2014-2018.

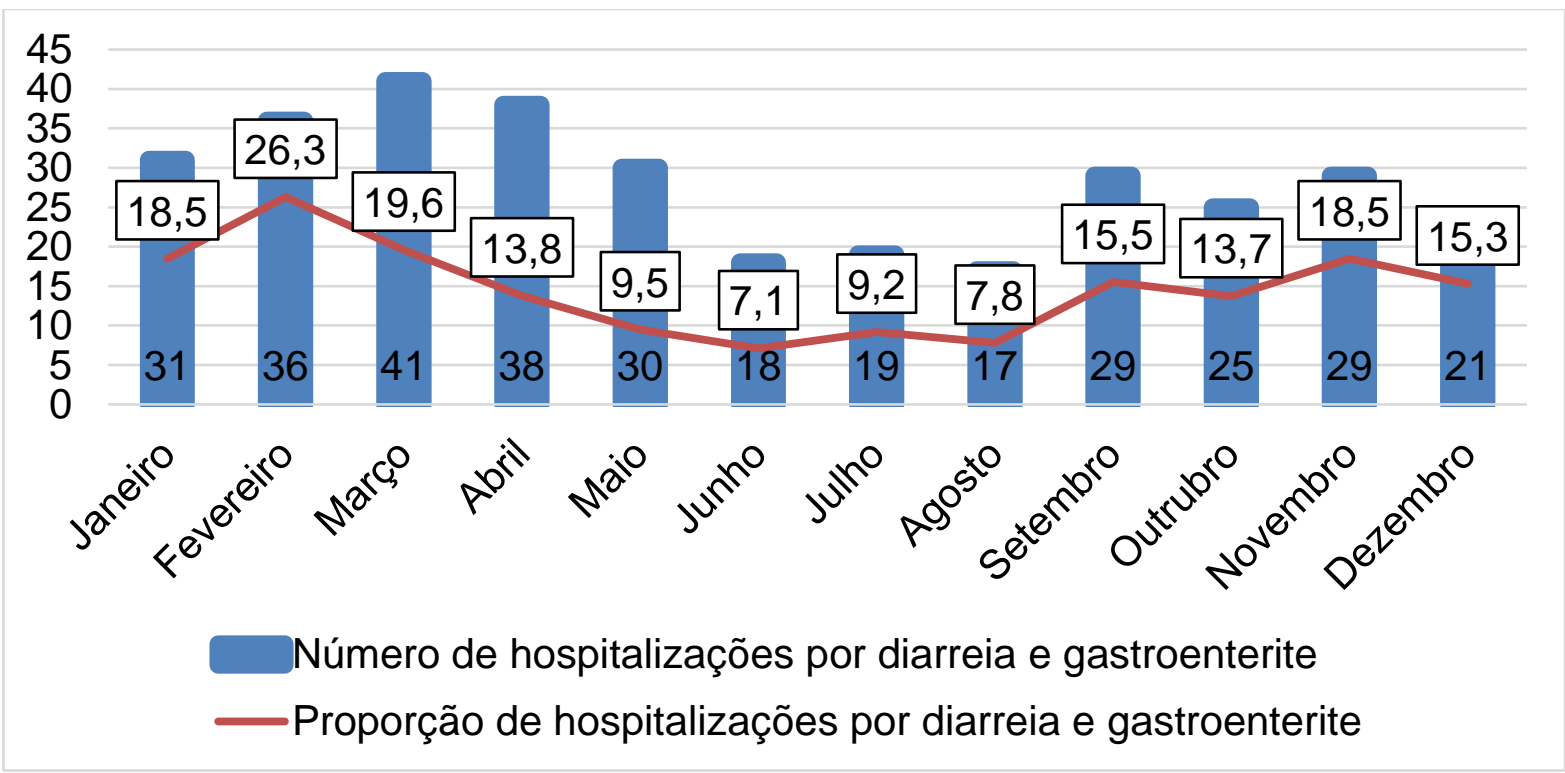

Fonte: Prontuário de Internamento. Hospital Universitário Júlio Bandeira, CajazeirasPB: 01 de janeiro de 2014 a 31 de dezembro de 2018.

Em relação à zona de procedência dos pacientes, os residentes em zona urbana somaram 232 internamentos $(69,5 \%)$ e os advindos da zona rural, 102 internamentos $(30,5 \%)$.

Vinte municípios foram referidos como local de residência dos pacientes, dentre eles, a cidade de Cajazeiras-PB somou o maior número de internações, 190 (56,9\%). Dos 15 municípios que compõem a 9a Região de Saúde da Paraíba, o único que não registrou internamentos no serviço foi o munícipio de Joca ClaudinoPB. Contudo, cinco municípios, os quais não fazem parte da 9a Região, registraram internamentos, foram eles: 'Ibiara-PB', 'Pocinhos-PB', 'Serra Grande-PB', 'João Pessoa-PB' e 'Nazarezinho-PB'.

A Tabela 3, a seguir, demonstra a distribuição das internações por diarreia e gastroenterite por municípios de residência. 
Tabela 3 - Distribuição do número de internamentos devido à diarreia e gastroenterite por município de residência - Hospital Universitário Júlio Bandeira, Cajazeiras-PB, 2014-2018.

\begin{tabular}{lcc}
\hline Municípios de Residência & N & $\%$ \\
\hline Cajazeiras & 190 & 56,9 \\
São José de Piranhas & 30 & 9,0 \\
São João do Rio do Peixe & 20 & 6,0 \\
Cachoeira dos Índios & 16 & 4,8 \\
Bernardino Batista & 10 & 3,0 \\
Triunfo & 10 & 3,0 \\
Uiraúna & 10 & 3,0 \\
Santa Helena & 9 & 2,7 \\
Monte Horebe & 8 & 2,4 \\
Poço Dantas & 7 & 2,1 \\
Poço de José de Moura & 6 & 1,8 \\
Bom Jesus & 4 & 1,2 \\
Carrapateira & 4 & 1,2 \\
Santarém & 3 & 0,9 \\
Serra Grande & 2 & 0,6 \\
Bonito de Santa Fé & 1 & 0,3 \\
lbiara & 1 & 0,3 \\
João Pessoa & 1 & 0,3 \\
Nazarezinho & 1 & 0,3 \\
Pocinhos & 1 & 0,3 \\
Total & 334 & 100,0 \\
\hline
\end{tabular}

Fonte: Prontuário de Internamento. Hospital Universitário Júlio Bandeira, CajazeirasPB: 01 de janeiro de 2014 a 31 de dezembro de 2018.

A faixa de idade dos pacientes variou de 1 mês a 18 anos, obtendo-se uma média de idade de 3,3 anos. As crianças menores de 5 anos destacaram-se por somar 244 hospitalizações, representando $73,1 \%$ do total. No Gráfico 1, é possível observar a distribuição do número de internações de acordo com as faixas de idade dos pacientes. 
Gráfico 1 - Distribuição do número de internamentos devido à diarreia e gastroenterite de acordo com as faixas de idade dos pacientes - Hospital Universitário Júlio Bandeira, Cajazeiras-PB, 2014-2018.

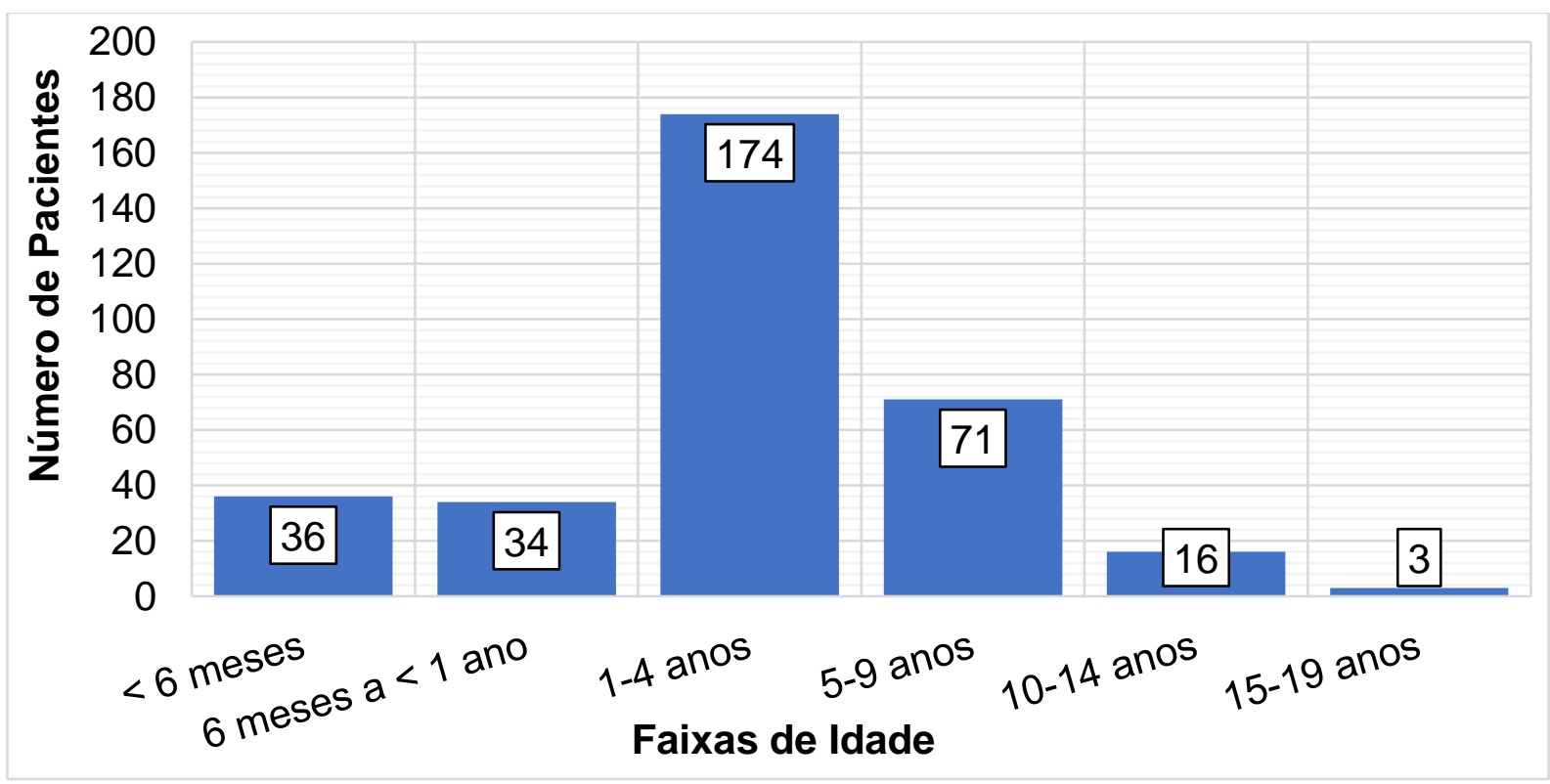

Fonte: Prontuário de Internamento. Hospital Universitário Júlio Bandeira, CajazeirasPB: 01 de janeiro de 2014 a 31 de dezembro de 2018

As crianças de cor/raça parda totalizaram 193 internações $(57,8 \%)$, cor/raça branca com $93(27,8 \%)$, preta com 4 internações $(1,2 \%)$ e amarela com $2(0,6 \%)$. Em outros 42 registros, a cor/raça dos pacientes não foi informada, cerca de 12,6\%. Por fim, 187 internações $(56,0 \%)$ corresponderam aos pacientes do sexo masculino e $147(44,0 \%)$ do sexo feminino.

\section{DISCUSSÃO}

Ao longo dos anos da pesquisa, percebeu-se redução no número de internações em crianças e adolescentes por diarreia e gastroenterite neste serviço. Pesquisas desenvolvidas a nível nacional também evidenciaram essa tendência, como o estudo de Moura et al. (2010), que ao analisar as causas de hospitalização 
em menores de 20 anos, constataram que a taxa de internação por gastroenterites infecciosas e suas complicações reduziu $12 \%$ entre os anos de 1999 a 2006 . E Rodrigues-Bastos et al. (2013), por sua vez, identificaram que a taxa de hospitalização por gastroenterite reduziu 19,5\%, ao comparar-se aos períodos compreendidos entre 2002-2005 e 2006-2009.

A redução mais expressiva da proporção de hospitalizações por diarreia e gastroenterite no serviço em relação à redução numérica pode ter ocorrido devido ao impacto crescente de outras patologias na morbidade de crianças e adolescentes. Esse fenômeno pode ser observado no estudo de Moura et al. (2010), o qual, ao analisarem as principais causas de hospitalizações por condições sensíveis à atenção básica em menores de 20 anos entre os anos de 1999 a 2006, detectaram uma redução de $13,1 \%$ nas taxas de internação por gastroenterites infecciosas e complicações na região Nordeste, ao passo que as taxas de internação por pneumonia cresceram 166,9\%.

Nesse sentido, os reais motivos que podem ter influenciado na diminuição do número de internações no Hospital Universitário Júlio Bandeira não foram contemplados como objetivos do estudo. Entretanto, supõe-se que os municípios atendidos pelo serviço possam ter alcançado, no período em estudo, um aumento da cobertura e/ou da qualidade dos serviços de saneamento (água tratada, rede coletora de esgoto e serviço de coleta de lixo) e que a população possa ter adquirido melhores condições habitacionais e/ou socioeconômicas, maior informação em saúde, acesso aos serviços de saúde, entre outros.

Segundo estudos, baixos índices de cobertura dos serviços de saneamento, condições habitacionais inapropriadas, baixa renda familiar, menor informação em saúde, menor cobertura do serviço de Atenção Primária à Saúde, entre outros, são implicados como importantes fatores de risco para o desenvolvimento de doenças diarreicas e gastroenterite (PAZ; ALMEIDA; GÜNTHER, 2012; MONAHAN et al., 2013).

O estudo de Wiegering et al. (2011), identificou que as maiores proporções de internamentos por diarreia em crianças ocorreram também nos primeiros meses do ano, especialmente entre janeiro e maio. Além disso, é importante ressaltar que os estudos publicados acerca desse tema frequentemente divergem em relação aos 
números mensais dos internamentos por doenças diarreicas, uma vez que a apresentação das doenças infecciosas intestinais pode ser influenciada por fatores geográficos, climáticos e sociodemográficos, os quais são específicos de cada local onde a pesquisa ocorreu.

Destaca-se também que a maioria dos pacientes são da zona urbana. Proporção similar foi encontrada por Thompson et al. (2015), que encontraram um percentual de $81,6 \%$ dos pacientes residentes em zona urbana. Pode-se supor que os maiores números tenham ocorrido nessa população por esta representar maior proporção relativa, visto que 76,8 \% dos domicílios no estado da Paraíba localizamse em zona urbana (IBGE, 2010). Já quanto ao maior número de hospitalizações registrado pela cidade de Cajazeiras-PB, pode ser justificado pelo fato de ser 0 município sede do Hospital Universitário, onde ocorreu o estudo e, assim, facilitar o acesso ao serviço, além de ser também o município mais populoso dessa microrregião de saúde.

Os indivíduos com idade inferior a cinco anos somaram a maior parte das internações no Hospital Universitário Júlio Bandeira, destacando-se a faixa de idade de 1 a 4 anos. Outros estudos conduzidos com crianças hospitalizadas por diarreia, também registraram proporções semelhantes, como o de Meneguessi et al. (2015), no qual foi identificado que crianças de 1 a 4 anos somaram $57,3 \%$ das admissões.

Os maiores números identificados nos indivíduos de 1 a 4 anos, podem ser explicados porque essa população não detém noções adequadas de higiene, como lavar as mãos após defecação, por exemplo, além do ato de engatinhar e/ou brincar no solo ou em áreas potencialmente contaminadas por fezes de animais ou humanos, visto que é nessa fase que as crianças ganham maior independência, o que favorece a contaminação por patógenos (PEDRAZA; QUEIROZ; SALES, 2014).

Além disso, é estimado que o episódio diarreico em crianças menores de cinco anos tenha a probabilidade de ser severo cerca de dez vezes maior do que em crianças maiores ou em adultos, o que também pode favorecer maiores números de hospitalizações em relação a esses indivíduos (LAMBERTI; WALKER; BLACK, 2012).

Também chama a atenção o elevado percentual de hospitalizações registrado por crianças menores de um ano de idade. Esse grupo foi dividido na presente 
pesquisa em $<6$ meses e $>6$ meses e, ambas as faixas de idade registraram números bastante próximos entre si. Essa divisão foi realizada, pois de acordo com o Ministério da Saúde, deve-se realizar amamentação exclusiva até os 6 meses de idade e, após esse período, recomenda-se a introdução de outros alimentos na dieta da criança (BRASIL, 2003).

Fatores como erro alimentar e precariedade de higiene familiar podem ter influenciado o registro desses números de hospitalizações em $<1$ ano de idade, pois segundo a literatura, a adesão ao aleitamento materno consiste em um fator de proteção aos indivíduos de menor idade e práticas adequadas de higiene familiar, como o preparo correto e condicionamento do alimento das crianças, evitam a contaminação alimentar e o desenvolvimento dessas patologias nas crianças (MOTA et al., 2015; SERGIO; LEON, 2009).

Além disso, percebeu-se uma redução do número de admissões hospitalares com o aumento da faixa etária da amostra, visto que os indivíduos de 10-14 anos somaram $4,8 \%$ e os de $15-19$ anos, apenas $0,9 \%$. Esse resultado pode estar associado ao fato de que o risco de desenvolver diarreia diminui com o aumento da idade, como identificado por Pinzón-Rondón et al. (2015). Outros possíveis motivos podem ser a maior noção de higiene e de formas de prevenção de doenças, além de que, com o aumento da idade há também aquisição da imunidade por rotavírus, que é uma das principais etiologias das doenças diarreicas em crianças (WALKER; SACK; BLACK, 2010).

Fontoura et al. (2018), também identificaram que a prevalência e incidência de doenças diarreicas foi maior nos indivíduos de cor/raça parda. Esse resultado pode ter sido encontrado porque a cor/raça parda é a de maior representatividade na população do estado da Paraíba, que soma cerca de 53,2\% (IBGE, 2010).

Outros estudos em crianças internadas por doenças infecciosas intestinais encontraram maiores proporções de pacientes do sexo masculino, alcançando $52,2 \%$ e 54,4\% da amostra (CHUNG et al., 2017; LIND et al., 2016). Tal fato pode estar relacionado com fatores socioculturais, como maior tendência à realização de atividades fora do ambiente domiciliar e assim, consequentemente, proporcionar maior exposição a esses patógenos. 


\section{CONCLUSÃO}

A redução do número de hospitalizações por diarreia, gastroenterite ou doenças infecciosas intestinais no Hospital Universitário Júlio Bandeira ao longo do período de estudo traz esperança. Entretanto, por serem patologias passíveis de prevenção através de medidas básicas de promoção da saúde, esse ainda é um cenário bastante preocupante para a região.

A identificação do período do ano que ocorre maior número dessas hospitalizações e das características epidemiológicas desses pacientes pode favorecer a elaboração de planos estratégicos para o Hospital Universitário Júlio Bandeira, tendo por objetivo melhorar a qualidade da atenção à saúde prestada, a exemplo de aquisição de insumos específicos para o tratamento e condução clínica desses pacientes em período oportuno, além de treinamento e atualização profissional acerca do tema.

Esse conhecimento pode nortear também campanhas de educação em saúde em âmbito comunitário, a fim de evitar o surgimento e a propagação dessas doenças antes do período esperado de aumento da demanda hospitalar.

É válido também ressaltar que os dados da pesquisa advêm de informações contidas nos prontuários médicos, estando, portanto, sujeitos à incompletude de informações. 


\section{REFERÊNCIAS BIBLIOGRÁFICAS}

ANDERS, K. L. et al. The epidemiology and aetiology of diarrhoeal disease in infancy in southern Vietnam: a birth cohort study. International Journal of Infectious Diseases, v. 35, p. 3-10, 2015.

ARAÚJO, T. M. E. de et al. Surto de diarreia por rotavírus no município de Bom Jesus (PI). Revista Ciência \& Saúde Coletiva, v. 15, n. 1, p. 1039-1046, 2010.

BRASIL. Ministério da Saúde. Dez passos para uma alimentação saudável: guia alimentar para crianças menores de 2 anos: álbum seriado / Ministério da Saúde, - Brasília, 2003.

BÜHLER, H. F. et al. Spatial analysis of integrated health and environmental indicators for morbidity and mortality due to infant diarrhea in Brazil, 2010. Cadernos de Saúde Pública, v. 30, n. 9, p. 1921-1934, 2014.

CHUNG, N.et al. Clinical and epidemiological characteristics in hospitalized young children with acute gastroenteritis in southern Taiwan: According to major pathogens. Journal of Microbiology, Immunology and Infection, v. 50, n. 6, p. 915-922, 2017.

Disponível em: http://www2.ebserh.gov.br/documents/1132097/4032000/PDE+20192020/89fa552b-40f4-4729-9472-f798b0655779?version=1.1. Acesso em: 08/07/2019.

FONTOURA, V. M. et al. Socio-environmental factors and diarrheal diseases in under five-year old children in the state of Tocantins, Brazil. PloSone, v. 13, n. 5, p. e0196702, 2018.

Instituto Brasileiro de Geografia e Estatística (IBGE). Censo Demográfico, 2010. Domicílios particulares permanentes e moradores em domicílios particulares permanentes, por situação do domicílio, segundo as mesorregiões, as microrregiões e os municípios Paraíba - 2010.2 Disponível em: ftp://ftp.ibge.gov.br/Censos/Censo_Demografico_2010/Familias_e_Domicilios/Xls/Unidades_da_ Federacao/paraiba_xls.zip >. Acesso em 23 jul. 2019.

Instituto Brasileiro de Geografia e Estatística (IBGE). Censo Demográfico, 2010. Pessoas em famílias únicas e conviventes principais residentes em domicílios particulares, por cor ou raça, segundo a situação do domicílio e a condição na família - Paraíba - 2010. Disponível em:

ftp://ftp.ibge.gov.br/Censos/Censo_Demografico_2010/Familias_e_Domicilios/xls/Unidades_da_ Federacao/paraiba_xls.zip >. Acesso em 24 jul. 2019.

LAMBERTI, L. M.; WALKER, C. L. F.; BLACK, R. E. Systematic review of diarrhea duration and severity in children and adults in low-and middle-income countries. BMC public health, v. 12, n. 1, p. 276, 2012.

LIND, C. H. et al. Variation in diagnostic testing and hospitalization rates in children with acute gastroenteritis. Hospital pediatrics, v. 6, n. 12, p. 714-721, 2016.

MENEGUESSI, G. M. et al. Morbimortalidade por doenças diarreicas agudas em crianças menores de 10 anos no Distrito Federal, Brasil, 2003 a 2012. Epidemiologia e Serviços de Saúde, v. 24, n. 3, p. 721-730, 2015.

MONAHAN, L. J. et al. Impact of the Family Health Program on gastroenteritis in children in Bahia, Northeast Brazil: an analysis of primary care-sensitive conditions. Journal of epidemiology and global health, v. 3, n. 3, p. 175-185, 2013. 
MOTA, T. T. A. G. et al. Influência do aleitamento materno na hospitalização de menores de dois anos no estado de Pernambuco, Brasil, em 1997 e 2006. Ciência \& Saúde Coletiva, v. 20, n. 8, p. 2347-2358, 2015.

MOURA, B. L. A. et al. Principais causas de internação por condições sensíveis à atenção primária no Brasil: uma análise por faixa etária e região. Rev. Bras. Saúde Matern. Infant., v. 10, n. Suplemento 1, p. S83-S91, 2010.

PAZ, M. G. A. da; ALMEIDA, M. F. de; GÜNTHER, W. M. R. Prevalência de diarreia em crianças e condições de saneamento e moradia em áreas periurbanas de Guarulhos, SP. Revista Brasileira de Epidemiologia, v. 15, n. 1, p. 188-197, 2012.

PEDRAZA, D. F.; QUEIROZ, D. de; SALES, M. C. Doenças infecciosas em crianças préescolares brasileiras assistidas em creches. Ciência \& Saúde Coletiva, v. 19, p. 511-528, 2014.

PINZÓN-RONDÓN, Á. M. et al. Country characteristics and acute diarrhea in children from developing nations: a multilevel study. BMC public health, v. 15, n. 1, p. 811, 2015.

RODRIGUES-BASTOS, R. M. et al. Internações por condições sensíveis à atenção primária em município do sudeste do Brasil. Revista da Associação Médica Brasileira, v. 59, n. 2, p. 120 127, 2013.

SERGIO, J. V.; LEON, A. C. P. de. Analysis of mortality from diarrheic diseases in under-five children in Brazilian cities with more than 150,000 inhabitants. Cadernos de Saúde Pública, v. 25, n. 5, p. 1093-1102, 2009.

SILVA, E. N. et al. Plano Diretor Estratégico do Hospital Universitário Júlio Bandeira de Mello, da Universidade Federal de Campina Grande-HUJB/UFCG. Biênio 2019-2020.

THOMPSON, C. N. et al. A prospective multi-center observational study of children hospitalized with diarrhea in Ho Chi Minh City, Vietnam. The American journal of tropical medicine and hygiene, v. 92, n. 5, p. 1045-1052, 2015.

WALKER, C. L. F.; BLACK, R. E. Zinc for the treatment of diarrhoea: effect on diarrhoea morbidity, mortality and incidence of future episodes. International journal of epidemiology, $v$. 39, n. suppl_1, p. i63-i69, 2010.

WIEGERING, V. et al. Gastroenteritis in childhood: a retrospective study of 650 hospitalized pediatric patients. International Journal of Infectious Diseases, v. 15, n. 6, p. e401-e407, 2011.

WIELGOS, K. et al. Management of acute gastroenteritis in children. Pol Med J, v. XLVII, n. 278, p. 76-79, 2019.

WORLD HEALTH ORGANIZATION. Physical Status: The use and interpretation of Anthopometry. Genova, 1995. 